\title{
Clinical Comparison of Pulse and Chirp Excitation
}

\author{
Pedersen, Morten Høgholm; Misaridis, T.; Jensen, Jørgen Arendt
}

Published in:

IEEE Ultrasonics Symposium, 2002. Proceedings

Link to article, DOI:

10.1109/ULTSYM.2002.1192617

Publication date:

2002

Document Version

Publisher's PDF, also known as Version of record

Link back to DTU Orbit

Citation (APA):

Pedersen, M. H., Misaridis, T., \& Jensen, J. A. (2002). Clinical Comparison of Pulse and Chirp Excitation. In IEEE Ultrasonics Symposium, 2002. Proceedings (pp. 1632-1635). IEEE.

https://doi.org/10.1109/ULTSYM.2002.1192617

\section{General rights}

Copyright and moral rights for the publications made accessible in the public portal are retained by the authors and/or other copyright owners and it is a condition of accessing publications that users recognise and abide by the legal requirements associated with these rights.

- Users may download and print one copy of any publication from the public portal for the purpose of private study or research.

- You may not further distribute the material or use it for any profit-making activity or commercial gain

- You may freely distribute the URL identifying the publication in the public portal

If you believe that this document breaches copyright please contact us providing details, and we will remove access to the work immediately and investigate your claim. 


\title{
Clinical Comparison of Pulse and Chirp Excitation*
}

\author{
Morten H. Pedersen ${ }^{1,2}$, Thanassis X. Misaridis ${ }^{1, \dagger}$ and Jørgen A. Jensen ${ }^{1}$ \\ ${ }^{1}$ Center for Fast Ultrasound Imaging, Ørsted•DTU, Bldg. 348, Technical University of Denmark, \\ DK-2800 Kgs. Lyngby, Denmark \\ ${ }^{2}$ Department of Ultrasound, Herlev Hospital, University of Copenhagen, \\ DK-2730 Herlev, Denmark
}

\begin{abstract}
Coded excitation (CE) using frequency modulated signals (chirps) combined with modified matched filtering has earlier been presented showing promising results in simulations and in-vitro. In this study an experimental ultrasound system is evaluated in a clinical setting, where image sequences are assessed by skilled medical doctors. The effect on penetration depth and image quality were measured. A modified clinical scanner with a $4 \mathrm{MHz}$ single element mechanical transducer, and external transmitter and receiver boards (RASMUS system) were used. The system allowed rapid toggling between chirp and short pulse excitation to simultaneously produce identical image sequences using both techniques. Nine healthy male volunteers were scanned in abdominal locations. All sequences were evaluated by 3 skilled medical doctors, blinded to each other and to the technique used. They assessed the depth 1) in which image quality decreased and 2) in which the image would be insufficient for clinical diagnosis. Furthermore they compared image quality in matching pairs of conventional and CE images. The average increase in penetration depth were almost $2 \mathrm{~cm}$. Side-by-side comparison showed that coded image quality was consistently rated better; significant $(\mathrm{p} \leq 0.05)$ when images were cropped at minimum the depth for good image quality and highly significant $(\mathrm{p}<0.001)$ when cropped at maximum depth sufficient for clinical diagnosis. We conclude that coded excitation with linear FM chirps improves penetration
\end{abstract}

\footnotetext{
*Work supported by grant 9700883 and 9700563 from the Danish Science Foundation and B-K Medical A/S. Thanks to M.D. Dr.Med. Torben Larsen, M.D. Nis Nørgaard, M.D. Bjørn Skjoldbye, M.Sc. Kim Gammelmark, and Ph.D., M.Sc. Charlotte Hindsberger.

${ }^{\dagger}$ Thanassis Misaridis is currently at Laboratoire Ondes et Acoustique, E.S.P.C.I., 10 rue Vauquelin, 75005 Paris, France
}

and image quality significantly in a clinical setting.

\section{INTRODUCTION}

There has been an increasing interest in utilizing more sophisticated excitation signals, than the single-carrier short pulses currently used in ultrasound scanners, to increase the signal-to-noise ratio (SNR). Higher SNR will allow imaging of structures located deeper inside the human body or alternatively allow migration to higher frequencies, which in turn will result in images with better resolution. This way either penetration or image resolution can be gained without loosing the other.

The aim of this study was to evaluate the performance of coded excitation in-vivo. The following two null hypotheses were tested: 1) Coded excitation has no effect on penetration depth and 2) Coded excitation has no effect on image quality.

\section{Material AND Methods}

Nine healthy male volunteers were scanned in supine position by an experienced sonographer (MHP). Mean age was 32.6 (from 25.5 to 42.5 ) years, mean weight 76.9 (from 65 to 93 ) $\mathrm{kg}$ and mean body mass index (BMI) was 23.5 (from 20.1 to 27.7). Three different views were scanned in each of the nine persons (Table 1), yielding 54 cine-loop sequences (27 paired sequences). At each location an interleaved sequence of 2 seconds (30 frames) duration was recorded.

A modified clinical ultrasound scanner (Type 3535, B-K Medical A/S, Herlev, Denmark) was used with a mechanical transducer (Model 8534, $4 \mathrm{MHz}$ pivoting focused piston type). External transmitter and receiver 


\begin{tabular}{cl}
\hline No. & Location \\
\hline 1 & $\begin{array}{l}\text { Sub-costal transverse section of right liver lobe. } \\
2\end{array}$ \\
& $\begin{array}{l}\text { Sub-costal saggital section of right liver lobe in- } \\
\text { cluding right kidney. }\end{array}$ \\
3 & $\begin{array}{l}\text { Epigastric transverse section of liver pointing to the } \\
\text { right depicting the right liver lobe. }\end{array}$ \\
\hline
\end{tabular}

Table 1: Scanning locations.

boards were both developed and produced at CFU as a part of our Remotely Accessible Software-configurable Multi-channel Ultrasound System (RASMUS) [1]. The transmit signal was amplified using a power RF amplifier (RITEC 5000) designed to drive ultrasound transducers. Amplification and time-gain compensation were done by the scanner before sampling by the receiver board (12 bits at $40 \mathrm{MHz}$ ). B-mode display on the scanner allowed orientation of the transducer before and during acquisition.

The transmitter's ability to rapidly toggle between different pulse types during scanning were used to record pulsed and coded images interleaved. Hereby, every second frame was pulsed and coded respectively, providing images of the exact same location being directly comparable. The recording was done at 13 frames per second (fps), yielding 6.5 fps of each kind.

The short pulse used was a $1 \frac{1}{2}$-cycle Hanning weighted cosine at $4 \mathrm{MHz}$ with a $65 \%$ fractional bandwidth $(-3 \mathrm{~dB})$. The coded waveform was a linear FM signal sweeping a fractional bandwidth of $110 \%$. The signal was shaped using a Tukey window with a duration of 0.15 times pulse duration [2], resulting in a transmitted signal with a fractional $\mathrm{BW}$ of $65 \%$ like the short pulse.

Intensity measurements were carried out using a calibrated hydrophone in a water-tank. Standard intensities and mechanical index were far below the recommended maximum values (Table 2).

All data processing were done using MATLAB ${ }^{\circledR}$ (MathWorks Inc., Natick, Mass., USA). Both coded and pulsed data were compressed by the appropriate filter to maximize SNR. The short pulse signal was compressed using a matched filter, the chirp using the mismatched filter described in [2], which is the linear FM signal weighted using a Chebyshev window with a $80 \mathrm{~dB}$ sidelobe level. Then envelope detection (Hilbert transform

\begin{tabular}{rlll}
\hline \multicolumn{1}{c}{ Pulsed } & Coded & \\
\hline In water & & & \\
$I_{\text {sptp }}$ & 12 & 25 & $\mathrm{~W} / \mathrm{cm}^{2}$ \\
$I_{\text {sppa }}$ & 3.0 & 1.8 & $\mathrm{~W} / \mathrm{cm}^{2}$ \\
$I_{\text {spta }}$ & 0.019 & 0.46 & $\mathrm{~mW} / \mathrm{cm}^{2}$ \\
\hline In situ & & & \\
$I_{\text {sptp }}$ & 1.8 & 3.6 & $\mathrm{~W} / \mathrm{cm}^{2}$ \\
$I_{\text {sppa }}$ & 0.43 & 0.26 & $\mathrm{~W} / \mathrm{cm}^{2}$ \\
$I_{\text {spta }}$ & $2.8 \cdot 10^{-3}$ & $6.6 \cdot 10^{-2}$ & $\mathrm{~mW} / \mathrm{cm}^{2}$ \\
\hline MI & 0.08 & 0.12 & \\
\hline
\end{tabular}

Table 2: Measured ultrasound intensities.

followed by absolute value) and log-compression were carried out.

The TGC was corrected before scan-line conversion using automatic post-processing based on statistical properties of the recorded data (method described in [3]).

Every image sequence was converted to movie clips (AVI-files using lossless compression - Huffyuv CODEC v2.1.1 $1^{1}$ ) for evaluation on an ordinary PC. Three experienced sonographers (medical doctors) evaluated the cine-loops. None of them were otherwise involved in the project, nor had they seen any of the images beforehand. Evaluations were done blinded and independently of each other.

All 54 sequences ( 27 pulsed and 27 coded) were presented in random order to each sonographer with no information on the type of technique used.

For each, two questions were to be answered :

- Question 1: At what depth [cm] does the image quality decrease significantly?

- Question 2: At what depth [cm] does the image quality become insufficient for clinical diagnosis?

To compare the image quality of coded and conventional imaging, matching pairs of image sequences recorded at the exact same location were showed simultaneously side-by-side in random order, with the conventional or coded image randomly placed to the left to avoid bias from potential left-right preferences or expectancies in the examiners.

\footnotetext{
${ }^{1}$ http://www.math.berkeley.edu/ $/$ benrg/huffyuv.html
} 


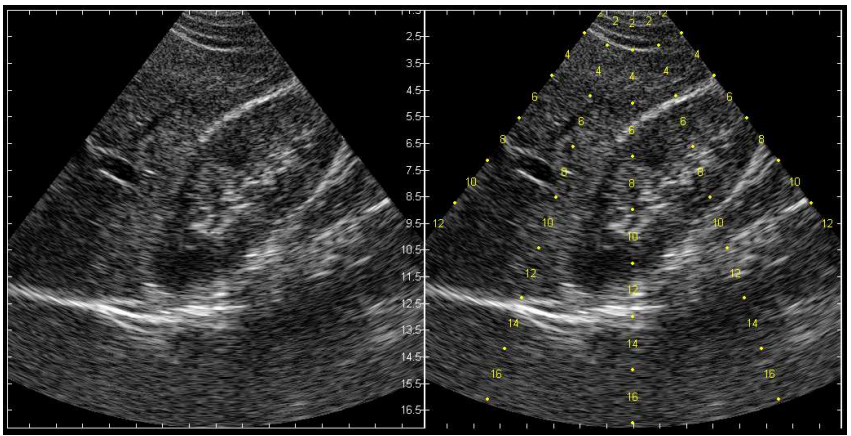

Figure 1: Layout of cine-loop presentation of single images. Images are identical except for the rulers for depth estimation.

For each of the 27 image pairs, two cine-loops were created. The images were cropped below the depths: $d_{\text {magiq }}$ and $d_{\text {maui }}$ calculated for each image pair from the answers to Questions 1 and 2:

$$
\begin{aligned}
& d_{\text {magiq }}=\min \left(\frac{\sum_{n=1}^{N} d_{n, Q_{1}, C}}{N}, \frac{\sum_{n=1}^{N} d_{n, Q_{1}, P}}{N}\right) \\
& d_{\text {maui }}=\max \left(\frac{\sum_{n=1}^{N} d_{n, Q_{2}, C}}{N}, \frac{\sum_{n=1}^{N} d_{n, Q_{2}, P}}{N}\right)
\end{aligned}
$$

where $N$ is the total number of examiners and $d_{1, Q_{2}, C}$ means examiner one's answer to Question 2 for the coded image. The first depth $\left(d_{\text {magiq }}\right)$ represents the "minimum average good image quality" depth for the image pair. The second $\left(d_{\text {maui }}\right)$ represents the "maximum average usable image" depth. Comparison of the images cropped at $d_{\text {magiq }}$ therefore only evaluates image quality within a range, where both techniques should provide good image quality according to the sonographers' evaluation.

Comparison of images cut at the second depth $d_{\text {maui }}$ evaluates image quality within the maximum usable range of that pair judged by the sonographers. In this case, images produced by the technique with best penetration are expected to do best.

This distinction was made to evaluate, not only if codes could improve image quality by increasing penetration depth $\left(d_{\text {maui }}\right)$, but also if it provides the same image quality at the range readily obtainable by conventional pulsed ultrasound imaging $\left(d_{\text {magiq }}\right)$.

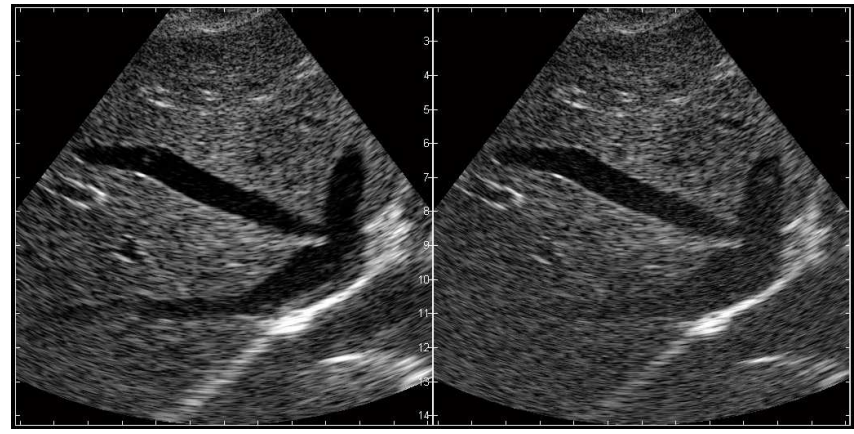

Figure 2: Presentation of image pair cine-loops. In this case the left is codede and the right is a conventional image.

For each of the 54 pairs the sonographers were asked which of the two images was better (Fig. 2) by scoring on a visual analog scale (Fig. 3). To avoid attraction to the divisions of the scale only the line without numbers and explanations were shown during the actual scoring of each image pair.
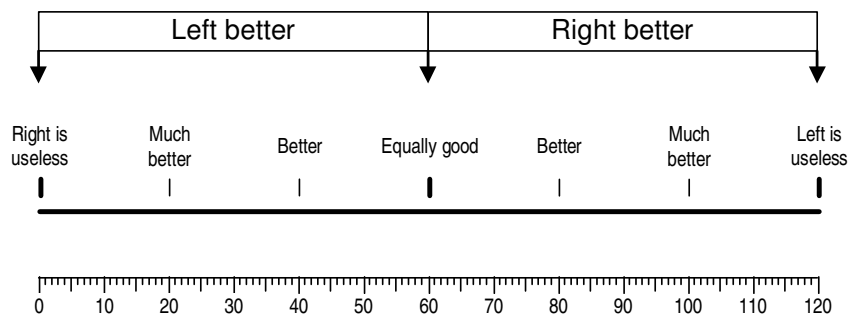

Figure 3: Visual analog scale used for image comparison.

The data analysis language $\mathrm{R}$ (http://cran.rproject.org/) was used for statistical computations. Student's (one sample) t-test was used on the resulting differences in penetration depth, assuming normal distribution (supported by the data). Two-sided tests were used. No assumptions of normal distributed data were made about side-by-side image comparisons. Consequently, Wilcoxon signed rank test with continuity correction was used on VAS results.

\section{RESULTS}

The resulting coded ultrasound images were generally good, with less noise and better penetration than corresponding conventional images.

The penetration differences between coded and con- 
ventional imaging are listed in Table 3. All three examiners found a highly significant increase in imaging depths. The average increase in penetration depth was $1.98 \mathrm{~cm}$ [examiner range $0.7-3 \mathrm{~cm}$ ] in question 1 and $1.85 \mathrm{~cm}$ [examiner range 1.2-2.2 cm] in question 2 .

In images cropped at $d_{\text {magiq }}$ the difference was just barely there, though significantly in favor of coded imaging, with examiner mean values from 1.61 to 3.48 on the VAS ranging from -60.0 to 60.0 (Table 4).

The difference was more pronounced in images cropped at $d_{\text {maui }}$ with mean scores $18.5,13.9$, and 5.87 respectively; highly significant, in favor of coded imaging (Table 4).

\section{Discussion}

The experimental setup allowing simultaneous recording of both coded and conventional pulsed excitation images, provided a good platform for a direct comparison of paired image sequences acquired under the exact same circumstances.

To our best knowledge clinical evaluation of coded excitation has not been reported before (PubMed - Medline). The present work shows that coded excitation performs well in-vivo. No severe artifacts except repeating echoes before and after very strong specular reflectors, such as the diaphragm, were encountered. We believe this problem will be solved using optimized receive amplifiers.

Blinded evaluation by medical doctors showed increased penetration and image quality using $\mathrm{CE}$. As predicted by previous simulations and in-vitro studies, CE significantly increased the imaging depth in a clinical setting with almost $2 \mathrm{~cm}$. Furthermore, images done using CE significantly preferred for clinical diagnosis by the doctors - even when cut off at penetration depths where conventional images begins to degrade.

The clinical benefit of increased penetration is obvious to the sonographer that daily experiences cases with insufficient penetration resulting in diagnostic uncertainty. Alternatively, a frequency increase yielding higher resolution whilst maintaining penetration is also appealing. The possible improvement of diagnosis, treatment and prognosis, though, remains to be tested in randomized controlled trials.

\begin{tabular}{|c|c|c|c|c|}
\hline \multicolumn{5}{|c|}{ Question 1} \\
\hline Examiner & $\begin{array}{c}\text { Pulsed } \\
{[\mathrm{cm}]}\end{array}$ & $\begin{array}{c}\text { Coded } \\
{[\mathrm{cm}]}\end{array}$ & $\begin{array}{l}\text { Diff. } \\
{[\mathrm{cm}]}\end{array}$ & $\begin{array}{c}\mathrm{P} \\
\text { value }\end{array}$ \\
\hline S1 & 5.63 & 8.65 & 3.02 & $<0.001$ \\
\hline S2 & 8.85 & 9.59 & 0.741 & $<0.005$ \\
\hline S3 & 8.80 & 11.0 & 2.17 & $<0.001$ \\
\hline Pooled & 7.76 & 9.73 & 1.98 & $<0.001$ \\
\hline \multicolumn{5}{|c|}{ Question 2} \\
\hline Examiner & $\begin{array}{c}\text { Pulsed } \\
{[\mathrm{cm}]}\end{array}$ & $\begin{array}{c}\text { Coded } \\
{[\mathrm{cm}]}\end{array}$ & $\begin{array}{l}\text { Diff. } \\
{[\mathrm{cm}]}\end{array}$ & $\begin{array}{c}\mathrm{P} \\
\text { value }\end{array}$ \\
\hline S1 & 8.91 & 11.1 & 2.24 & $<0.001$ \\
\hline S2 & 11.6 & 12.8 & 1.19 & $<0.001$ \\
\hline S3 & 10.4 & 12.6 & 2.13 & $<0.001$ \\
\hline Pooled & 10.3 & 12.2 & 1.85 & $<0.001$ \\
\hline
\end{tabular}

Table 3: Results of answers to Questions "At what depth [cm] does the image quality (1) decrease significantly and (2) become insufficient for clinical diagnosis?"

\begin{tabular}{ccr}
\hline Sonographer & \multicolumn{2}{c}{ Difference } \\
& $d_{\text {MAGIQ }}$ & $d_{M A U I}$ \\
\hline $\mathrm{S} 1$ & $3.31^{* *}$ & $18.5^{* * *}$ \\
$\mathrm{~S} 2$ & $1.61^{*}$ & $13.9^{* * *}$ \\
$\mathrm{~S} 3$ & $1.72^{\dagger}$ & $5.87^{* *}$ \\
\hline ***: $\mathrm{P}<0.001,{ }^{* *}: \mathrm{P}<0.005, *: \mathrm{P}<0.05, \dagger: \mathrm{P}=0.05$
\end{tabular}

Table 4: Mean VAS differences by examiner for images cut at $d_{M A U I}$ and $d_{M A G I Q}$. Positive values: coded is better.

\section{$\mathrm{V}$ REFERENCES}

[1] J. A. Jensen, O. Holm, L. J. Jensen, H. Bendsen, H. M. Pedersen, K. Salomonsen, J. Hansen, and S. Nikolov. Experimental ultrasound system for real-time synthetic imaging. In Proc. IEEE Ultrason. Symp., volume 2, pages 1595-1599, 1999.

[2] T. X. Misaridis and J. A. Jensen. An effective coded excitation scheme based on a predistorted fm signal and an optimized digital filter. In IEEE Ultrasonics Symposium Proceedings, volume 2, pages 1589-1593, 1999.

[3] M. H. Pedersen, T. X. Misaridis, and J. A. Jensen. Clinical evaluation of coded excitation in medical ultrasound. submitted to Ultrasound in Medicine \& Biology, 2002. 